\title{
Measurement of the $t$-channel single top quark production cross section
}

\section{DØ Collaboration}

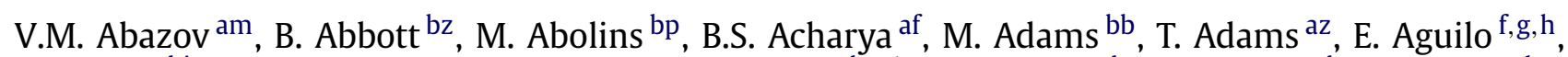
M. Ahsan bj, G.D. Alexeev am, G. Alkhazov ${ }^{\mathrm{aq}}$, A. Alton ${ }^{\mathrm{bo}, 1}{ }^{\text {, G. Alverson }}{ }^{\text {bn }}$, G.A. Alves ${ }^{\mathrm{b}}$, L.S. Ancu ${ }^{\text {al }}$, M.S. Anzelc ${ }^{\text {bd }}$, M. Aoki ${ }^{\text {ba }}$, Y. Arnoud $^{\mathrm{p}}, \mathrm{M}$. Arov ${ }^{\text {bk }}$, M. Arthaud ${ }^{\mathrm{t}}$, A. Askew $^{\mathrm{az}, 2}$, B. Åsman $^{\text {ar,as }}$, O. Atramentov ${ }^{\mathrm{az}, 2}$, C. Avila ${ }^{\mathrm{j}}$, J. BackusMayes ${ }^{\mathrm{cg}}$, F. Badaud $^{\mathrm{O}}$, L. Bagby ${ }^{\text {ba }}$, B. Baldin ${ }^{\text {ba }}$, D.V. Bandurin ${ }^{\text {bj }}$, S. Banerjee af ${ }^{\text {a }}$ E. Barberis ${ }^{\text {bn }}$, A.-F. Barfuss ${ }^{\text {q }}$, P. Bargassa ${ }^{\text {ce }}$, P. Baringer ${ }^{\text {bi }}$, J. Barreto ${ }^{\text {b }}$, J.F. Bartlett ${ }^{\text {ba }}$, U. Bassler ${ }^{\mathrm{t}}$, D. Bauer au, S. Beale ${ }^{\mathrm{f}, \mathrm{g}, \mathrm{h}}$, A. Bean ${ }^{\text {bi }}$, M. Begalli ${ }^{\mathrm{c}}$, M. Begel $^{\text {bx }}$, C. Belanger-Champagne $^{\text {ar, as }}$, L. Bellantoni ${ }^{\text {ba }}$, A. Bellavance ${ }^{\text {ba }}$, J.A. Benitez ${ }^{\text {bp }}$, S.B. Beri ${ }^{\text {ad }}$, G. Bernardi ${ }^{\text {s }}$, R. Bernhard ${ }^{\mathrm{y}}$, I. Bertram ${ }^{\text {at }}$, M. Besançon ${ }^{t}$, R. Beuselinck ${ }^{\text {au }}$, V.A. Bezzubov ${ }^{\text {ap }}$, P.C. Bhat ${ }^{\text {ba }}$, V. Bhatnagar ${ }^{\text {ad }}$, G. Blazey ${ }^{\text {bc }}$, S. Blessing ${ }^{\text {az }}$, K. Bloom ${ }^{\text {br }}$, A. Boehnlein ${ }^{\text {ba }}$, D. Boline ${ }^{\text {bm }}$, T.A. Bolton ${ }^{\text {bj }}$, E.E. Boos ${ }^{\text {ao }}$, G. Borissov $^{\text {at }}$, T. Bose $^{\text {bm }}$, A. Brandt $^{c c}$, R. Brock ${ }^{\text {bp }}$, G. Brooijmans ${ }^{\text {bu }}$, A. Bross ${ }^{\text {ba }}$, D. Brown ${ }^{\mathrm{u}}$, X.B. Bu ${ }^{\mathrm{i}}$, D. Buchholz ${ }^{\text {bd }}$, M. Buehler $^{\text {cf }}$, V. Buescher $^{\mathrm{X}}$, V. Bunichev ${ }^{\text {ao }}$, S. Burdin ${ }^{\text {at, } 3}$, T.H. Burnett ${ }^{c g}$, C.P. Buszello ${ }^{a u}$, P. Calfayan ${ }^{\mathrm{ab}}$, B. Calpas ${ }^{\mathrm{q}}$, S. Calvet ${ }^{\mathrm{r}}$, J. Cammin ${ }^{\text {bv }}$, M.A. Carrasco-Lizarraga aj, E. Carrera ${ }^{\mathrm{az}}$, W. Carvalho ${ }^{\mathrm{c}}$, B.C.K. Casey ${ }^{\text {ba }}$, H. Castilla-Valdez ${ }^{\text {aj }}$, S. Chakrabarti ${ }^{\text {bw }}$, D. Chakraborty ${ }^{\text {bc }}$, K.M. Chan ${ }^{\text {bf }}$, A. Chandra ${ }^{\text {ay }}$, E. Cheu ${ }^{\text {aw }}$, D.K. Cho ${ }^{\text {bm }}$, S.W. Cho ${ }^{\text {ah }}$, S. Choi ${ }^{\text {ai }}$, B. Choudhary ae, T. Christoudias au, S. Cihangir ${ }^{\text {ba }}$, D. Claes ${ }^{\text {br }}$, J. Clutter ${ }^{\text {bi }}$, Y. Coadou ${ }^{\text {f,g,h, }}$, M. Cooke ${ }^{\text {ba }}$, W.E. Cooper ${ }^{\text {ba }}$, M. Corcoran ${ }^{c e}$, F. Couderc ${ }^{t}$, M.-C. Cousinou ${ }^{\mathrm{q}}$, D. Cutts ${ }^{\mathrm{cb}}$, M. Ć Ciok $^{\mathrm{ag}}$, A. Das ${ }^{a w}$, G. Davies ${ }^{a u}$, K. De ${ }^{c c}$, S.J. de Jong ${ }^{a l}$, E. De La Cruz-Burelo ${ }^{\text {aj }}$, K. DeVaughan ${ }^{\text {br }}$, F. Déliot ${ }^{t}$, M. Demarteau ${ }^{\text {ba }}$, R. Demina ${ }^{\text {bv }}$, D. Denisov ${ }^{\text {ba }}$, S.P. Denisov ${ }^{\text {ap }}$, S. Desai ${ }^{\text {ba }}$, H.T. Diehl ${ }^{\text {ba }}$, M. Diesburg ${ }^{\text {ba }}$,

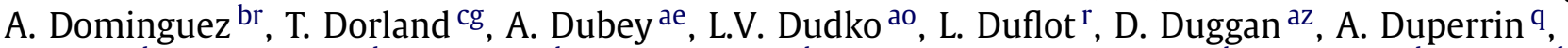
S. Dutt ${ }^{a d}$, A. Dyshkant ${ }^{\text {bc }}$, M. Eads ${ }^{\text {br }}$, D. Edmunds ${ }^{\text {bp }}$, J. Ellison ay , V.D. Elvira ba , Y. Enari ${ }^{c b}$, S. Eno $^{\mathrm{bl}}$, M. Escalier ${ }^{q}$, H. Evans $^{\text {be }}$, A. Evdokimov ${ }^{\text {bx }}$, V.N. Evdokimov ${ }^{\text {ap }}$, G. Facini ${ }^{\text {bn }}$, A.V. Ferapontov ${ }^{\text {bj }}$, T. Ferbel ${ }^{\text {bl, bv }}$, F. Fiedler aa ${ }^{\text {a F. Filthaut }}{ }^{\text {al }}$, W. Fisher ${ }^{\text {ba }}$, H.E. Fisk ${ }^{\text {ba }}$, M. Fortner ${ }^{\text {bc }}$, H. Fox ${ }^{\text {at }}$, S. Fu ${ }^{\text {ba }}$,

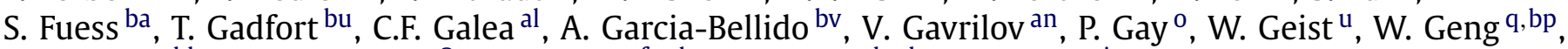
C.E. Gerber ${ }^{\text {bb }}$, Y. Gershtein ${ }^{\mathrm{az}, 2}$, D. Gillberg ${ }^{\mathrm{f}, \mathrm{g}, \mathrm{h}}$, G. Ginther ${ }^{\text {ba,bv }}$, B. Gómez ${ }^{\mathrm{j}}$, A. Goussiou $^{\mathrm{cg}}$, P.D. Grannis ${ }^{\text {bw }}$, S. Greder ${ }^{\mathrm{u}}$, H. Greenlee ${ }^{\text {ba }}$, Z.D. Greenwood ${ }^{\text {bk }}$, E.M. Gregores ${ }^{\mathrm{d}}$, G. Grenier ${ }^{\mathrm{v}}$, Ph. Gris ${ }^{\mathrm{o}}$, J.-F. Grivaz ${ }^{\text {r }}$, A. Grohsjean ${ }^{\text {t}}$, S. Grünendahl ${ }^{\text {ba }}$, M.W. Grünewald ${ }^{\text {ag }}$, F. Guo ${ }^{\text {bw }}$, J. Guo ${ }^{\text {bw }}$, G. Gutierrez ${ }^{\text {ba }}$, P. Gutierrez ${ }^{\text {bz }}$, A. Haas ${ }^{\text {bu }}$, P. Haefner ${ }^{\text {ab }}$, S. Hagopian ${ }^{a z}$, J. Haley ${ }^{\text {bs }}$, I. Hall ${ }^{\text {bp }}$, R.E. Hall ${ }^{\text {ax }}$, L. Han ${ }^{\text {i }}$, K. Harder ${ }^{\text {av }}$, A. Harel ${ }^{\text {bv }}$, J.M. Hauptman ${ }^{\text {bh }}$, J. Hays ${ }^{\text {au }}$, T. Hebbeker ${ }^{\text {w }}$, D. Hedin ${ }^{\text {bc }}$, J.G. Hegeman ${ }^{\text {ak }}$, A.P. Heinson ay , U. Heintz ${ }^{\text {bm }}$, C. Hensel $^{z}$, I. Heredia-De La Cruz ${ }^{\text {aj }}$, K. Herner ${ }^{\text {bo }}$, G. Hesketh $^{\text {bn }}$, M.D. Hildreth bf , R. Hirosky ${ }^{\text {cf }}$, T. Hoang ${ }^{\text {az }}$, J.D. Hobbs ${ }^{\text {bw }}$, B. Hoeneisen ${ }^{n}$, M. Hohlfeld ${ }^{\mathrm{x}}$, S. Hossain ${ }^{\text {bz }}$, P. Houben ${ }^{\text {ak }}$, Y. Hu ${ }^{\text {bw }}$, Z. Hubacek ${ }^{1}$, N. Huske ${ }^{\text {, }}$, V. Hynek ${ }^{1}$, I. Iashvili bt, R. Illingworth ${ }^{\text {ba }}$, A.S. Ito ${ }^{\text {ba }}$, S. Jabeen ${ }^{\text {bm }}$, M. Jaffré ${ }^{r}$, S. Jain ${ }^{\text {bz }}$, K. Jakobs ${ }^{\mathrm{y}}$, D. Jamin ${ }^{\mathrm{q}}$, R. Jesik ${ }^{\text {au }}$, K. Johns ${ }^{\mathrm{aw}}{ }^{\text {, C. Johnson }}{ }^{\text {bu }}$, M. Johnson ${ }^{\text {ba }}$, D. Johnston ${ }^{\text {br }}$, A. Jonckheere ${ }^{\text {ba }}$, P. Jonsson ${ }^{\text {au }}$, A. Juste ${ }^{\text {ba }}$, E. Kajfasz ${ }^{\mathrm{q}}$, D. Karmanov ${ }^{\text {ao }}$, P.A. Kasper ${ }^{\text {ba }}$, I. Katsanos ${ }^{\text {br }}$, V. Kaushik ${ }^{c c}$, R. Kehoe ${ }^{\text {cd }}$, S. Kermiche ${ }^{\text {q }}$, N. Khalatyan ba , A. Khanov ${ }^{\text {ca }}$, A. Kharchilava ${ }^{\text {bt }}$, Y.N. Kharzheev ${ }^{\text {am }}$, D. Khatidze ${ }^{\text {cb }}$, M.H. Kirby bd, M. Kirsch ${ }^{\text {w }}$, B. Klima ba J.M. Kohli ad, J.-P. Konrath ${ }^{y}$, A.V. Kozelov ap, J. Kraus bp, T. Kuhl ${ }^{\text {aa }}{ }^{\text {, A. Kumar }}{ }^{\text {bt }}$, A. Kupco ${ }^{\text {m, }}$, T. Kurča ${ }^{\text {v }}$, V.A. Kuzmin ${ }^{\text {ao }}$, J. Kvita $^{k}$, F. Lacroix ${ }^{\circ}$, D. Lam bf ${ }^{\text {bf }}$ S. Lammers ${ }^{\text {be }}$, G. Landsberg ${ }^{\text {cb }}$, P. Lebrun ${ }^{\text {v }}$, H.S. Lee ${ }^{\text {ah }}$, W.M. Lee ${ }^{\text {ba }}$, A. Leflat ${ }^{\text {ao }}$, J. Lellouch ${ }^{\text {s }}$, L. Li ${ }^{\text {ay }}$, Q.Z. Li ${ }^{\text {ba }}$, S.M. Lietti ${ }^{\text {e }}$, J.K. Lim ${ }^{\text {ah }}$, D. Lincoln ${ }^{\text {ba }}$, J. Linnemann ${ }^{\text {bp }}$, V.V. Lipaev ${ }^{\text {ap }}$, R. Lipton ${ }^{\text {ba }}$, Y. Liu ${ }^{i}$, Z. Liu ${ }^{\text {f,g,h }}$, A. Lobodenko ${ }^{\text {aq }}$, M. Lokajicek ${ }^{m}$, P. Love ${ }^{\text {at }}$, H.J. Lubatti $^{\text {cg }}$, 
R. Luna-Garcia ${ }^{\text {aj }, 5}$, A.L. Lyon ${ }^{\text {ba }}$, A.K.A. Maciel ${ }^{\text {b }}$, D. Mackin ${ }^{\text {ce }}$, P. Mättig ac, R. Magaña-Villalba aj, P.K. Mal ${ }^{\text {aw }}$, S. Malik ${ }^{\text {br }}$, V.L. Malyshev ${ }^{\text {am }}$, Y. Maravin ${ }^{\text {bj }}$, B. Martin ${ }^{\text {P }}$, R. McCarthy ${ }^{\text {bw }}$, C.L. McGivern ${ }^{\text {bi }}$, M.M. Meijer ${ }^{\text {al }}$, A. Melnitchouk ${ }^{\text {bq }}$, L. Mendoza ${ }^{j}$, D. Menezes ${ }^{\text {bc }}$, P.G. Mercadante ${ }^{e}$, M. Merkin $^{\text {ao }}$, K.W. Merritt ${ }^{\text {ba }}$, A. Meyer ${ }^{\mathrm{w}}$, J. Meyer ${ }^{\mathrm{z}}$, N.K. Mondal ${ }^{\text {af }}$, R.W. Moore ${ }^{\mathrm{f}, \mathrm{g}, \mathrm{h}}$, T. Moulik $^{\mathrm{bi}}$, G.S. Muanza ${ }^{\mathrm{q}}$, M. Mulhearn ${ }^{\text {bu }}$,

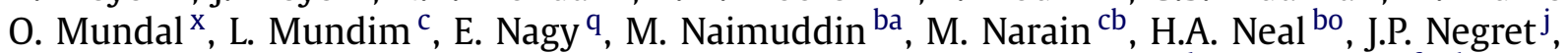
P. Neustroev ${ }^{\mathrm{aq}}$, H. Nilsen ${ }^{\mathrm{y}}$, H. Nogima ${ }^{\mathrm{c}}$, S.F. Novaes ${ }^{\mathrm{e}}$, T. Nunnemann ${ }^{\mathrm{ab}}$, D.C. O’Neil ${ }^{\mathrm{f}, \mathrm{g}, \mathrm{h}}$, G. Obrant ${ }^{\mathrm{aq}}$, C. Ochando ${ }^{r}$, D. Onoprienko ${ }^{\text {bj }}$, J. Orduna ${ }^{\text {aj }}$, N. Oshima ${ }^{\text {ba }}$, N. Osman ${ }^{\text {au }}$, J. Osta $^{\text {bf }}$, R. Otec ${ }^{l}$, G.J. Otero y Garzón a , M. Owen av, M. Padilla ay, P. Padley ${ }^{\mathrm{ce}}$, M. Pangilinan ${ }^{\mathrm{cb}}$, N. Parashar ${ }^{\text {bg }}$, S.-J. Park ${ }^{\mathrm{z}}$, S.K. Park ${ }^{\text {ah }}$, J. Parsons ${ }^{\text {bu }}$, R. Partridge ${ }^{c b}$, N. Parua be ${ }^{\text {, A. Patwa }}{ }^{b x}$, B. Penning ${ }^{\mathrm{y}}$, M. Perfilov ${ }^{\text {ao }}$, K. Peters ${ }^{\text {av }}$, Y. Peters ${ }^{a v}$, P. Pétroff ${ }^{r}$, R. Piegaia ${ }^{a}$, J. Piper ${ }^{b p}$, M.-A. Pleier ${ }^{\mathrm{x}}$, P.L.M. Podesta-Lerma aj,6, V.M. Podstavkov ${ }^{\text {ba }}$, Y. Pogorelov bf, M.-E. Pol ${ }^{\text {b }}$, P. Polozov an, A.V. Popov ap, M. Prewitt ${ }^{\text {ce }}$, H.B. Prosper ${ }^{\text {az }}$, S. Protopopescu ${ }^{\text {bx }}$, J. Qian ${ }^{\text {bo }}$, A. Quadt ${ }^{z}$, B. Quinn ${ }^{\text {bq }}$, A. Rakitine ${ }^{\text {at }}$, M.S. Rangel ${ }^{\mathrm{r}}$, K. Ranjan ${ }^{\text {ae }}$, P.N. Ratoff ${ }^{\text {at }}$, P. Renkel ${ }^{\text {cd }}$, P. Rich ${ }^{a v}$, M. Rijssenbeek ${ }^{\text {bw }}$, I. Ripp-Baudot ${ }^{\mathrm{u}}$, F. Rizatdinova ${ }^{\mathrm{ca}}$, S. Robinson ${ }^{\mathrm{au}}$, M. Rominsky ${ }^{\mathrm{bz}}$, C. Royon ${ }^{\mathrm{t}}$, P. Rubinov ${ }^{\text {ba }}$, R. Ruchti bf , G. Safronov an, G. Sajot ${ }^{\text {P }}$, A. Sánchez-Hernández ${ }^{\text {aj }}$, M.P. Sanders ${ }^{\text {ab }}$, B. Sanghi ${ }^{\text {ba }}$, G. Savage ${ }^{\text {ba }}$, L. Sawyer ${ }^{\text {bk }}$, T. Scanlon au, D. Schaile ${ }^{\text {ab }}$, R.D. Schamberger ${ }^{\text {bw }}$, Y. Scheglov aq, H. Schellman bd, T. Schliephake ac , S. Schlobohm ${ }^{c g}$, C. Schwanenberger ${ }^{\text {av }}$, R. Schwienhorst bp, *, J. Sekaric az, H. Severini bz, E. Shabalina ${ }^{\text {z }}$, M. Shamim ${ }^{\text {bj }}$, V. Shary ${ }^{t}$, A.A. Shchukin ${ }^{\text {ap }}$, R.K. Shivpuri ${ }^{\text {ae }}$, V. Siccardi ${ }^{\text {u }}$, V. Simak ${ }^{1}$, V. Sirotenko ba, P. Skubic bz, P. Slattery bv, D. Smirnov bf , G.R. Snow ${ }^{\text {br }}{ }^{\text {, J. Snow }}{ }^{\text {by }}$, S. Snyder ${ }^{\text {bx }}$, S. Söldner-Rembold av, L. Sonnenschein ${ }^{\text {w }}$, A. Sopczak ${ }^{\text {at }}$, M. Sosebee ${ }^{c c}$, K. Soustruznik ${ }^{k}$, B. Spurlock ${ }^{c c}$, J. Stark $^{\text {p }, ~ V . ~ S t o l i n ~}{ }^{\text {an }}$, D.A. Stoyanova ${ }^{\text {ap }}$, J. Strandberg bo, M.A. Strang ${ }^{\text {bt }}$, E. Strauss ${ }^{\text {bw }}$, M. Strauss ${ }^{\text {bz }}$, R. Ströhmer ${ }^{\text {ab }}$, D. Strom ${ }^{\text {bb }}$, L. Stutte ${ }^{\text {ba }}$, S. Sumowidagdo az, P. Svoisky al, M. Takahashi av, A. Tanasijczuk ${ }^{a}$, W. Taylor ${ }^{\mathrm{f}, \mathrm{g}, \mathrm{h}}$, B. Tiller $^{\mathrm{ab}}$, M. Titov $^{\mathrm{t}}$, V.V. Tokmenin ${ }^{\mathrm{am}}$, I. Torchiani $^{\mathrm{y}}$, D. Tsybychev ${ }^{\mathrm{bw}}$, B. Tuchming ${ }^{\mathrm{t}}$, C. Tully ${ }^{\text {bs }}$, P.M. Tuts ${ }^{\text {bu }}$, R. Unalan ${ }^{\text {bp }, ~ L . ~ U v a r o v ~}{ }^{\text {aq }}$, S. Uvarov ${ }^{\text {aq }}$, S. Uzunyan ${ }^{\text {bc }}$, P.J. van den Berg ${ }^{\text {ak }}$, R. Van Kooten ${ }^{\text {be, W.M. van Leeuwen }}{ }^{\mathrm{ak}}$, N. Varelas ${ }^{\text {bb }}$, E.W. Varnes ${ }^{\text {aw }}$, I.A. Vasilyev ${ }^{\mathrm{ap}}$, P. Verdier ${ }^{\mathrm{v}}$, L.S. Vertogradov ${ }^{\mathrm{am}}$, M. Verzocchi ${ }^{\text {ba }}$, M. Vesterinen ${ }^{\mathrm{av}}$, D. Vilanova ${ }^{\mathrm{t}}$, P. Vint ${ }^{\mathrm{au}}$, P. Vokac ${ }^{1}$, R. Wagner $^{\mathrm{bs}}$, H.D. Wahl ${ }^{\text {az }}$, M.H.L.S. Wang ${ }^{\text {bv }}$, J. Warchol ${ }^{\text {bf }}$, G. Watts ${ }^{\text {cg }}$, M. Wayne ${ }^{\text {bf }}$, G. Weber ${ }^{\text {aa }}$, M. Weber ${ }^{\text {ba, }}$, L. Welty-Rieger $^{\text {be }}$, A. Wenger ${ }^{y}, 8$, M. Wetstein ${ }^{\text {bl }}$, A. White ${ }^{\text {cc }}$, D. Wicke ${ }^{\text {ad }}$, M.R.J. Williams ${ }^{\text {at }}$, G.W. Wilson $^{\text {bi }}$, S.J. Wimpenny ${ }^{\text {ay }}$, M. Wobisch ${ }^{\text {bk }}$, D.R. Wood ${ }^{\text {bn }}$, T.R. Wyatt ${ }^{\text {av }}$, Y. Xie $^{\text {cb }}$, C. Xu $^{\text {bo }}$, S. Yacoob ${ }^{\text {bd }}$, R. Yamada ba , W.-C. Yang av, T. Yasuda ${ }^{\text {ba }}$, Y.A. Yatsunenko ${ }^{\text {am }}$, Z. Ye ${ }^{\text {ba }}$, H. Yin ${ }^{\mathrm{i}}$, K. Yip ${ }^{\text {bx }}$, H.D. Yoo ${ }^{\text {cb }}$, S.W. Youn ${ }^{\text {ba }}$, J. Yu ${ }^{\text {cc }}$, C. Zeitnitz ${ }^{\text {ac }}$, S. Zelitch ${ }^{\text {cf }}$, T. Zhao ${ }^{\text {cg }}$, B. Zhou ${ }^{\text {bo }}$, J. Zhu ${ }^{\text {bw }}$, M. Zielinski bv, D. Zieminska be, L. Zivkovic ${ }^{\text {bu }}$, V. Zutshi ${ }^{\text {bc }}$, E.G. Zverev ${ }^{\text {ao }}$

\footnotetext{
a Universidad de Buenos Aires, Buenos Aires, Argentina

${ }^{\mathrm{b}}$ LAFEX, Centro Brasileiro de Pesquisas Físicas, Rio de Janeiro, Brazil

${ }^{\mathrm{c}}$ Universidade do Estado do Rio de Janeiro, Rio de Janeiro, Brazil

d Universidade Federal do ABC, Santo André, Brazil

e Instituto de Física Teórica, Universidade Estadual Paulista, São Paulo, Brazil

${ }^{\mathrm{f}}$ University of Alberta, Edmonton, Alberta, Canada

g Simon Fraser University, Burnaby, British Columbia, Canada

h York University, Toronto, Ontario, and McGill University, Montreal, Quebec, Canada

${ }^{\text {i } U n i v e r s i t y ~ o f ~ S c i e n c e ~ a n d ~ T e c h n o l o g y ~ o f ~ C h i n a, ~ H e f e i, ~ P e o p l e ' s ~ R e p u b l i c ~ o f ~ C h i n a ~}$

j Universidad de los Andes, Bogotá, Colombia

k Center for Particle Physics, Charles University, Faculty of Mathematics and Physics, Prague, Czech Republic

${ }^{1}$ Czech Technical University in Prague, Prague, Czech Republic

m Center for Particle Physics, Institute of Physics, Academy of Sciences of the Czech Republic, Prague, Czech Republic

${ }^{\mathrm{n}}$ Universidad San Francisco de Quito, Quito, Ecuador

${ }^{\circ}$ LPC, Université Blaise Pascal, CNRS/IN2P3, Clermont, France

p LPSC, Université Joseph Fourier Grenoble 1, CNRS/IN2P3, Institut National Polytechnique de Grenoble, Grenoble, France

q CPPM, Aix-Marseille Université, CNRS/IN2P3, Marseille, France

${ }^{\mathrm{r}}$ LAL, Université Paris-Sud, IN2P3/CNRS, Orsay, France

${ }^{s}$ LPNHE, IN2P3/CNRS, Universités Paris VI and VII, Paris, France

${ }^{\mathrm{t}}$ CEA, Irfu, SPP, Saclay, France

u IPHC, Université de Strasbourg, CNRS/IN2P3, Strasbourg, France

v IPNL, Université Lyon 1, CNRS/IN2P3, Villeurbanne, and Université de Lyon, Lyon, France

w III. Physikalisches Institut A, RWTH Aachen University, Aachen, Germany

x Physikalisches Institut, Universität Bonn, Bonn, Germany

${ }^{y}$ Physikalisches Institut, Universität Freiburg, Freiburg, Germany

${ }^{z}$ II. Physikalisches Institut, Georg-August-Universität Göttingen, Göttingen, Germany

aa Institut für Physik, Universität Mainz, Mainz, Germany

ab Ludwig-Maximilians-Universität München, München, Germany

ac Fachbereich Physik, University of Wuppertal, Wuppertal, Germany

ad Panjab University, Chandigarh, India

ae Delhi University, Delhi, India
} 
af Tata Institute of Fundamental Research, Mumbai, India

ag University College Dublin, Dublin, Ireland

${ }^{\text {ah }}$ Korea Detector Laboratory, Korea University, Seoul, Republic of Korea

ai SungKyunKwan University, Suwon, Republic of Korea

aj CINVESTAV, Mexico City, Mexico

ak FOM-Institute NIKHEF and University of Amsterdam/NIKHEF, Amsterdam, The Netherlands

al Radboud University Nijmegen/NIKHEF, Nijmegen, The Netherlands

am Joint Institute for Nuclear Research, Dubna, Russia

an Institute for Theoretical and Experimental Physics, Moscow, Russia

ao Moscow State University, Moscow, Russia

ap Institute for High Energy Physics, Protvino, Russia

aq Petersburg Nuclear Physics Institute, St. Petersburg, Russia

ar Stockholm University, Stockholm, Sweden

as Uppsala University, Uppsala, Sweden

at Lancaster University, Lancaster, United Kingdom

au Imperial College, London, United Kingdom

av University of Manchester, Manchester, United Kingdom

aw University of Arizona, Tucson, AZ 85721, USA

ax California State University, Fresno, CA 93740, USA

ay University of California, Riverside, CA 92521, USA

az Florida State University, Tallahassee, FL 32306, USA

ba Fermi National Accelerator Laboratory, Batavia, IL 60510, USA

bb University of Illinois at Chicago, Chicago, IL 60607, USA

bc Northern Illinois University, DeKalb, IL 60115, USA

bd Northwestern University, Evanston, IL 60208, USA

be Indiana University, Bloomington, IN 47405, USA

bf University of Notre Dame, Notre Dame, IN 46556, USA

bg Purdue University Calumet, Hammond, IN 46323, USA

bh Iowa State University, Ames, IA 50011, USA

bi University of Kansas, Lawrence, KS 66045, USA

bj Kansas State University, Manhattan, KS 66506, USA

${ }^{\mathrm{bk}}$ Louisiana Tech University, Ruston, LA 71272, USA

bl University of Maryland, College Park, MD 20742, USA

bm Boston University, Boston, MA 02215, USA

bn Northeastern University, Boston, MA 02115, USA

bo University of Michigan, Ann Arbor, MI 48109, USA

bp Michigan State University, East Lansing, MI 48824, USA

bq University of Mississippi, University, MS 38677, USA

br University of Nebraska, Lincoln, NE 68588, USA

bs Princeton University, Princeton, NJ 08544, USA

bt State University of New York, Buffalo, NY 14260, USA

bu Columbia University, New York, NY 10027, USA

bv University of Rochester, Rochester, NY 14627, USA

bw State University of New York, Stony Brook, NY 11794, USA

bx Brookhaven National Laboratory, Upton, NY 11973, USA

by Langston University, Langston, OK 73050, USA

bz University of Oklahoma, Norman, OK 73019, USA

ca Oklahoma State University, Stillwater, OK 74078, USA

cb Brown University, Providence, RI 02912, USA

cc University of Texas, Arlington, TX 76019, USA

cd Southern Methodist University, Dallas, TX 75275, USA

${ }^{c e}$ Rice University, Houston, TX 77005, USA

cf University of Virginia, Charlottesville, VA 22901, USA

cg University of Washington, Seattle, WA 98195, USA

\section{A R T I C L E I N F O}

\section{Article history:}

Received 24 July 2009

Received in revised form 5 November 2009

Accepted 16 November 2009

Available online 21 November 2009

Editor: L. Rolandi

PACS

14.65.Ha

12.15.Ji

13.85.Qk

\section{A B S T R A C T}

The D0 Collaboration reports direct evidence for electroweak production of single top quarks through the $t$-channel exchange of a virtual $W$ boson. This is the first analysis to isolate an individual single top quark production channel. We select events containing an isolated electron or muon, missing transverse energy, and two, three or four jets from $2.3 \mathrm{fb}^{-1}$ of $p \bar{p}$ collisions at the Fermilab Tevatron Collider. One or two of the jets are identified as containing a $b$ hadron. We combine three multivariate techniques optimized for the $t$-channel process to measure the $t$ - and $s$-channel cross sections simultaneously. We measure cross sections of $3.14_{-0.80}^{+0.94} \mathrm{pb}$ for the $t$-channel and $1.05 \pm 0.81 \mathrm{pb}$ for the $s$-channel. The measured $t$-channel result is found to have a significance of 4.8 standard deviations and is consistent with the standard model prediction.

\footnotetext{
* Corresponding author.

E-mail address: schwier@pa.msu.edu (R. Schwienhorst).

1 Visitor from Augustana College, Sioux Falls, SD, USA.

2 Visitor from Rutgers University, Piscataway, NJ, USA.

3 Visitor from The University of Liverpool, Liverpool, UK.
} 

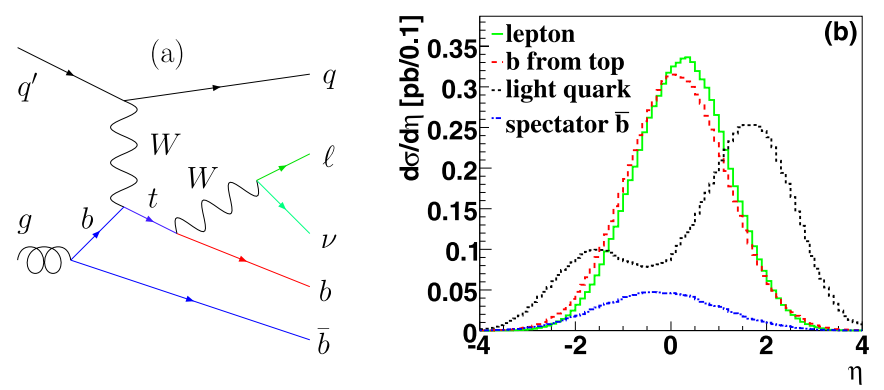

Fig. 1. Representative Feynman diagram for $t$-channel single top quark production and decay (a) and parton-level pseudorapidity distribution of the final state objects in top production (excluding antitop), requiring each object to have transverse momentum $>15 \mathrm{GeV}$ (b).

The $\mathrm{D} \varnothing$ and CDF Collaborations at the Fermilab Tevatron $p \bar{p}$ Collider have recently observed electroweak production of single top quarks [1,2], measuring the total single top quark production cross section as well as the Cabibbo-Kobayashi-Maskawa (CKM) matrix element [3] $\left|V_{t b}\right|$ directly. In the standard model (SM), the two main production modes at the Tevatron resulting in a single top (or antitop) quark final state are the $t$-channel exchange of a $W$ boson shown in Fig. $1 \mathrm{a}$ and the $s$-channel production via the decay of a virtual $W$ boson. The two observation analyses measured only the combined single top quark cross section, assuming the SM ratio of the two production modes. This ratio is modified in several new physics scenarios, for example in models with additional quark generations, new heavy bosons [4-7], flavor-changing neutral currents [8-10], or anomalous top quark couplings [11-15]. In this Letter we remove this assumption and use the $t$-channel characteristics to measure the $t$-channel and $s$-channel cross sections simultaneously, thus providing a $t$-channel measurement independent of the $s$-channel cross section model. The main characteristic of the $t$-channel which separates it both from the s-channel and the backgrounds is the pseudorapidity distribution of the light quark jet, shown in Fig. 1b. The predicted cross section for $t$-channel ( $s$-channel) production is $2.34 \pm 0.13 \mathrm{pb}$ $(1.12 \pm 0.04 \mathrm{pb})$ for a top quark mass $m_{t}=170 \mathrm{GeV}$ [16]

This analysis extends the Do single top evidence $[17,18]$ and observation analyses [1], utilizing the same dataset, event selection, and signal/background modeling as the observation analysis, but training multivariate filters specifically to extract $t$-channel single top quark events. We use $2.3 \mathrm{fb}^{-1}$ of data collected by the D0 experiment [19] at the Fermilab Tevatron $p \bar{p}$ Collider between 2002 and 2007 (Run II). The measurement selects final states containing one high transverse momentum $\left(p_{T}\right)$ isolated lepton (electron or muon), large missing transverse energy $\left(\mathbb{E}_{T}\right)$, a $b$ quark jet from the decay of the top quark ( $t \rightarrow W b \rightarrow \ell v b$ ), a light quark jet produced in association with the top quark, and a spectator $b$ quark jet from gluon splitting in the initial state. We allow for one of these jets not to be identified as well as for the presence of an additional jet from gluon radiation. The backgrounds are $W$ bosons produced in association with jets, $t \bar{t}$ pairs, and multijet production, where a jet is misreconstructed as an electron or a heavy-flavor quark decays to a muon that satisfies isolation criteria. $Z+$ jets and diboson processes form minor additional background components. We treat $s$-channel single top quark production as a background during the multivariate training but measure its cross section si-

\footnotetext{
4 Visitor from CERN, Geneva, Switzerland.

5 Visitor from Centro de Investigacion en Computacion - IPN, Mexico City, Mexico.

6 Visitor from ECFM, Universidad Autonoma de Sinaloa, Culiacán, Mexico.

7 Visitor from Universität Bern, Bern, Switzerland.

8 Visitor from Universität Zürich, Zürich, Switzerland.
}

Table 1

Number of expected and observed events in $2.3 \mathrm{fb}^{-1}$ for $e$ and $\mu$, and one and two $b$-tagged analysis channels combined, with uncertainties including both statistical and systematic components. The $t$-channel and $s$-channel contributions are normalized to their SM expectation.

\begin{tabular}{llll}
\hline Source & 2 jets & 3 jets & 4 jets \\
\hline$t$-channel & $77 \pm 10$ & $39 \pm 6$ & $14 \pm 3$ \\
$s$-channel & $62 \pm 9$ & $24 \pm 4$ & $7 \pm 2$ \\
$W+$ jets & $1829 \pm 161$ & $637 \pm 61$ & $180 \pm 18$ \\
$Z+$ jets and dibosons & $229 \pm 38$ & $85 \pm 17$ & $26 \pm 7$ \\
$t \bar{t} \rightarrow \ell \ell, \ell+$ jets & $222 \pm 35$ & $436 \pm 66$ & $484 \pm 71$ \\
Multijets & $196 \pm 50$ & $73 \pm 17$ & $30 \pm 6$ \\
Total prediction & $2615 \pm 192$ & $1294 \pm 107$ & $742 \pm 80$ \\
Data & 2579 & 1216 & 724 \\
\hline
\end{tabular}

multaneously with the $t$-channel measurement as explained below.

We look for $t$-channel and $s$-channel single top quark production in events with two to four jets with $p_{T}>15 \mathrm{GeV}$ and pseudorapidity $|\eta|<3.4$, with the leading jet additionally satisfying $p_{T}>25 \mathrm{GeV}$. We require $20<\not_{T}<200 \mathrm{GeV}$ for events with two jets and $25<\mathbb{E}_{T}<200 \mathrm{GeV}$ for events with three or four jets. Events must contain only one isolated electron with $p_{T}>15 \mathrm{GeV}$ and $|\eta|<1.1$ ( $p_{T}>20 \mathrm{GeV}$ for three- or four-jet events), or one isolated muon with $p_{T}>15 \mathrm{GeV}$ and $|\eta|<2.0$. The background from jets misidentified as leptons is kept to approximately $5 \%$ by requiring the total transverse energy of all final state objects $H_{T}\left(\ell, \not_{T}\right.$, jets) to be greater than 110 to $160 \mathrm{GeV}$, depending on the analysis channel, and by demanding that the $\mathbb{k}_{T}$ is not along the direction of the lepton or the leading jet in the transverse plane. To enhance the signal fraction, one or two of the jets are required to originate from $b$ hadrons, as implemented through a neural network (NN) $b$-jet tagging algorithm [20]. We divide the dataset into 24 independent analysis channels (separated by data taking period, lepton type, $b$-tag and jet multiplicity) and combine the results to maximize the signal sensitivity. Details on the selection criteria and background modeling are given in Ref. [18].

We generate $t$-channel and $s$-channel single top events with the SingleTop Monte Carlo (MC) generator [21]. The kinematics of the generated events closely match those predicted by next-to-leadingorder (NLO) calculations [22], particularly those including NLO corrections to the $t$-channel $2 \rightarrow 3$ process shown in Fig. 1a [23]. The ALPGEN leading-order MC event generator [24], interfaced to PYTHIA [25], is used to model $t \bar{t}, W+$ jets, and $Z+$ jets background events. We use the CTEQ6L1 parton distribution functions (CTEQ6M for single top) [26] and set the top quark mass to $170 \mathrm{GeV}$. We use GEANT [27] to simulate the response of the D0 detector to the MC events. The $t \bar{t}$ background is normalized to the predicted cross section [28]. The $Z+$ jets contributions are normalized to NLO cross sections [29]. The $W+$ jets background normalization, jet flavor composition, and jet angular distributions are obtained from data samples. We model the background from multijet production where a jet is misidentified as an isolated electron or muon using events from data containing lepton candidates which pass all of the lepton identification requirements except one, but otherwise resemble the signal events. We use PYTHIA to model diboson production.

We select 4519 lepton + jets events with at least one $b$-tagged jet, which are expected to contain $130 \pm 17$-channel $(93 \pm 14$ $s$-channel) single top events with an acceptance of $(2.5 \pm 0.3) \%$ $((3.7 \pm 0.5) \%)$. The expected sample composition is shown in Table 1.

Systematic uncertainties in the signal and background models are described in detail in Ref. [18]. The main uncertainties are due to the jet energy scale (JES) corrections and the tag-rate functions (TRF), with smaller contributions from MC statistics, correction for 

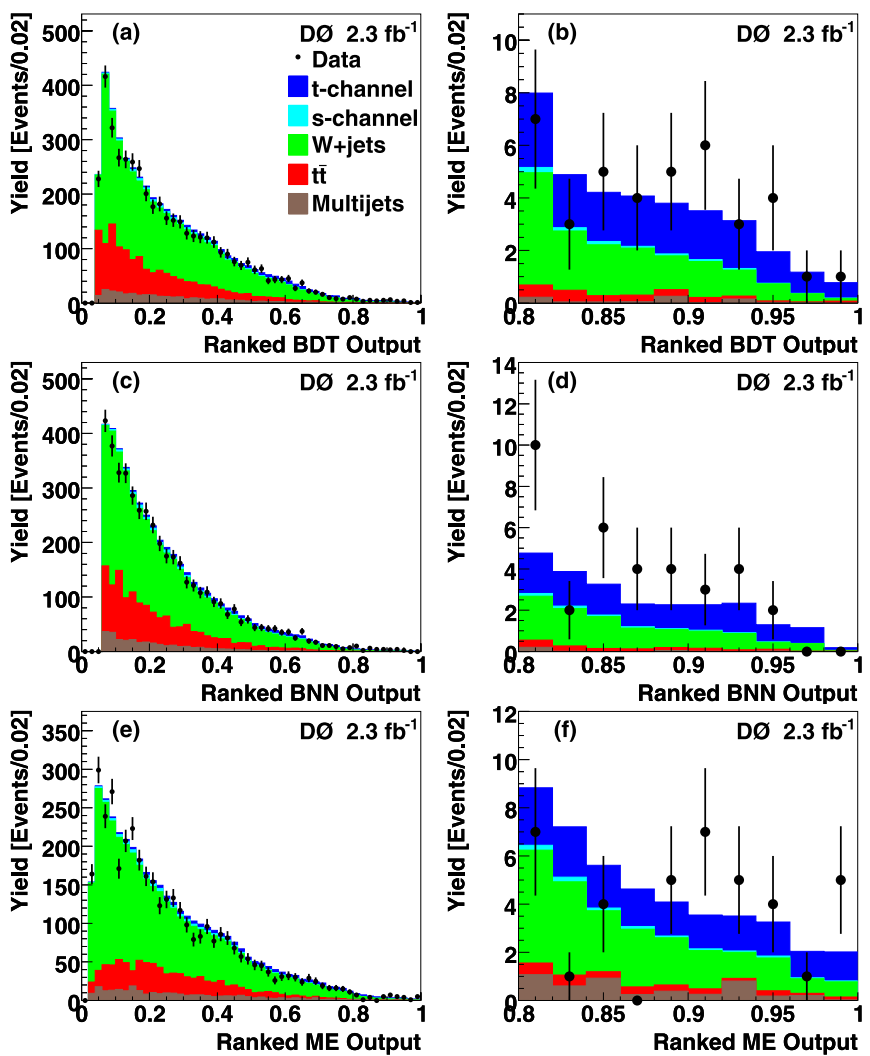

Fig. 2. Comparison of the signal and background models to data, for the BDT discriminant (a, b), the BNN discriminant (c, d), and the ME discriminant (e, f), for the full discriminant range $(a, c, e)$ and the signal region $(b, d, f)$. The bins have been ordered by their expected $t$-channel signal/background ratio and $t$-channel and $s$-channel single top distributions are normalized to the measured cross sections.

jet-flavor composition in $W+$ jets events, and from the $W+$ jets, multijets, and $t \bar{t}$ normalizations. The total uncertainty on the background is (8-16)\% depending on the analysis channel. Uncertainties on JES, TRFs and the modeling of $W+$ jets kinematics affect not only the normalization but also the shape of the discriminant distributions. Since the $W+$ jets background normalization and kinematics are constrained by data, theory prediction uncertainties for $W+$ jets are negligible (including those on the parton distribution functions and factorization and normalization scale).

We apply three independent multivariate analysis techniques to separate the small $t$-channel single top signal from the large backgrounds, based on boosted decision trees (BDT) [30-32], Bayesian neural networks (BNN) [33,34], and the matrix element (ME) method [35,36]. These techniques and their application are described in detail in Ref. [18]. For this analysis we use the same set of variables as in the observation paper [1]. However, only $t$-channel single top events are considered signal during the optimization, whereas $s$-channel single top events are included in the background, normalized to the SM expectation. Fig. 2 shows comparisons between the $t$-channel signal, the background model, and data for the three individual discriminants.

The three multivariate techniques use the same data sample but are not completely correlated. Their combination leads to increased sensitivity and a more precise measurement of the cross section. We achieve this by training a combination BNN which uses the three individual discriminant outputs as inputs. Fig. 3 shows the combination discriminant output for data superimposed on the background and signal models.
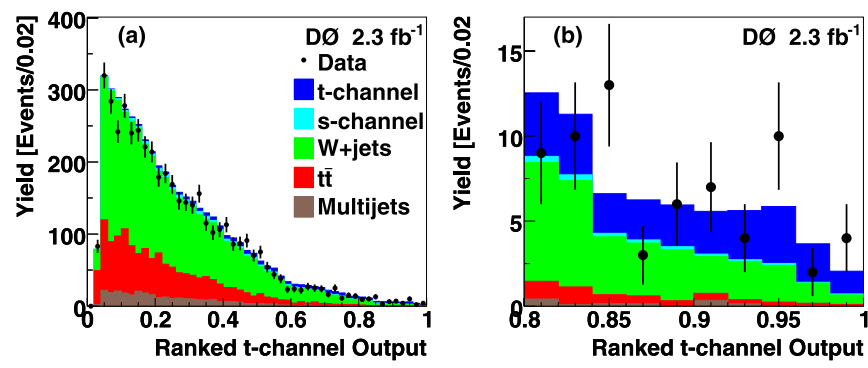

Fig. 3. Comparison of the signal and background models to data for the combination discriminant output, for the full range (a) and only the signal region (b). The bins have been ordered by their expected $t$-channel signal/background ratio and $t$-channel and $s$-channel single top distributions are normalized to the measured cross sections.
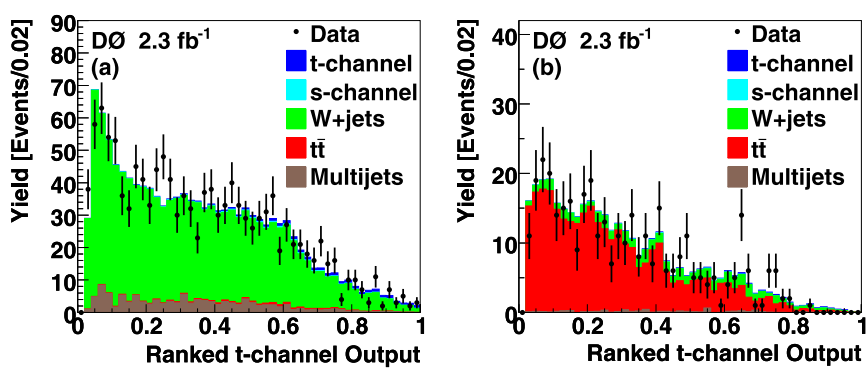

Fig. 4. Comparison of the background model to data for the ranked combination output, for a $W+$ jets (a) and a $t \bar{t}$ (b) dominated control sample.

We verify the accurate modeling of the data in backgrounddominated control regions for the two main background categories. Fig. 4a shows the $t$-channel discriminant in a $W+$ jets dominated sample of 2-jet, 1-tag events with $H_{T}<175 \mathrm{GeV}$. Fig. 4b shows the $t$-channel discriminant in a $t \bar{t}$ dominated sample of 4 -jet, 1-tag or 2-tag events with $H_{T}>300 \mathrm{GeV}$.

These studies confirm that backgrounds are well-modeled across the full range of the discriminant output.

We use a Bayesian statistical analysis [37] to measure the production cross sections. In a first step we compute the twodimensional posterior probability density as a function of both $t$-channel and $s$-channel single top quark cross sections. The combination discriminants for $t$-channel and $s$-channel single top, remaining background, and data are used to build a binned likelihood as a product over all analysis channels and bins. We assume a Poisson distribution for the observed counts, and flat prior probabilities for positive values of the $t$-channel and $s$-channel signal cross sections. Systematic uncertainties are described by Gaussian priors, and their correlations amongst all bins in all channels are preserved. The posterior probability density is shown in Fig. 5. Also shown are the SM expectation as well as several representative new physics models to illustrate the sensitivity of this analysis. Dedicated searches should be able to address flavor-changing neutral currents with a $Z$ boson coupling to the top and up quark with a strength of $4 \%$ of the SM coupling [4] or a top-color model with a $t \bar{b}$ bound state (Top Pion) with a mass of $m_{\pi}=250 \mathrm{GeV}$ [4], while a 4-quark-generations scenario with CKM matrix element $\left|V_{t s}\right|=0.2$ [38] or a top-flavor model with new heavy bosons at a scale $m_{x}=1 \mathrm{TeV}$ [4] will be more challenging to identify and might have to wait for LHC studies.

In a second step we obtain the $t$-channel posterior probability density from the two-dimensional posterior in Fig. 5 by integrating over the $s$-channel axis, thus not making any assumptions about the value of the $s$-channel cross section. We have analyzed ensembles of pseudo-datasets generated at several different $t$-channel 


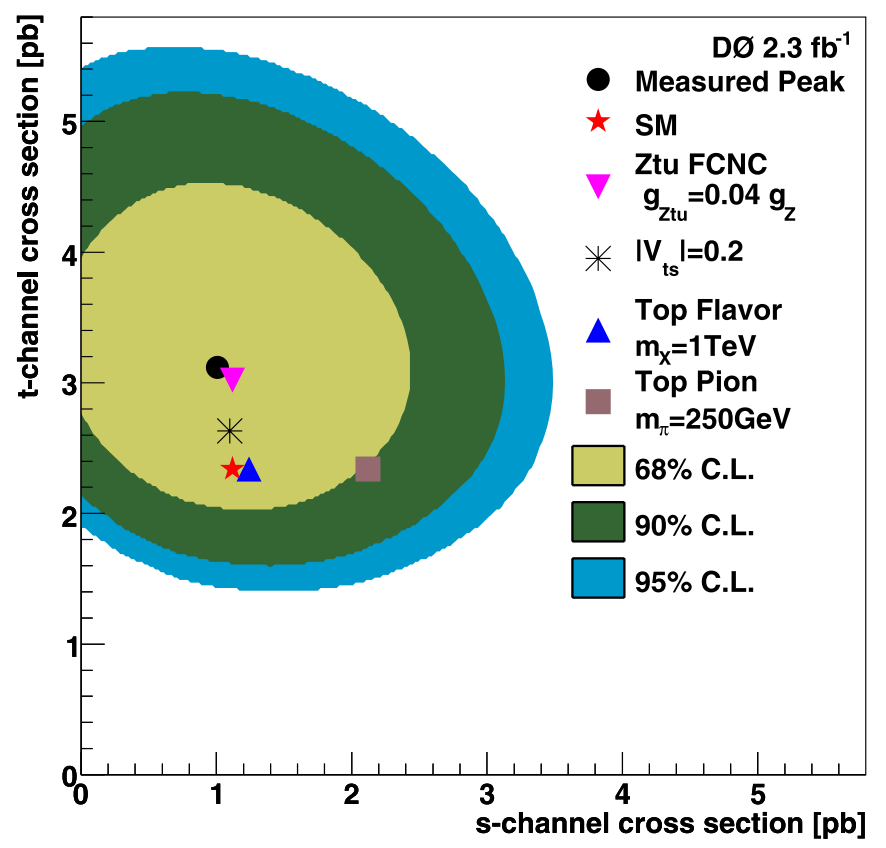

Fig. 5. Posterior probability density for $t$-channel and $s$-channel single top quark production in contours of equal probability density. Also shown are the measured cross section, SM expectation, and several representative new physics scenarios $[4,38]$.

and $s$-channel cross sections to verify the linearity of the measured $t$-channel cross section and its independence of the input $s$-channel cross section. From the $t$-channel posterior we extract the cross section and uncertainty for $t$-channel single top quark production as $3.14_{-0.80}^{+0.94} \mathrm{pb}$. We similarly extract the $s$-channel cross section as $1.05 \pm 0.81 \mathrm{pb}$ by integrating over the $t$-channel axis.

We compute the significance of the $t$-channel cross section measurement using pseudo-datasets generated from the background model (including SM $s$-channel single top) and taking all systematic uncertainties into account in a log-likelihood-ratio approach $[2,39]$. For each pseudo-dataset we calculate the ratio of the probabilities for two hypotheses: that the pseudo-dataset is described by the background model only (including SM s-channel), and that it is described by SM $t$-channel single top plus backgrounds. We measure the p-value by counting the fraction of background-only pseudo-datasets with a ratio that is more signallike than the one observed in data. The observed p-value is $8.0 \times$ $10^{-7}$, corresponding to a Gaussian significance of $4.8 \sigma$, and the expected p-value is $9.7 \times 10^{-5}$, corresponding to a Gaussian significance of $3.7 \sigma$.

We have checked the consistency of the observed signal with SM $t$-channel events in several kinematic distributions. Fig. 6 shows comparisons between the observed data, the background model, and the $t$-channel signal for four different kinematic distributions for events with a ranked discriminant output $>0.91$. Shown are four important kinematic variables for $t$-channel single top quark production: $H_{T}$; the reconstructed top quark mass; the lepton charge multiplied by the pseudorapidity of the leading non-b-tagged jet (cf. Fig. 1b); and the t-channel spin correlation in the optimal basis $[40,41]$, i.e. the cosine of the angle between the light quark jet and the lepton, both in the reconstructed top quark rest frame. While the background shapes resemble the signal in the high ranked discriminant output region, the presence of the $t$-channel signal is nevertheless clearly evident in each distribution.
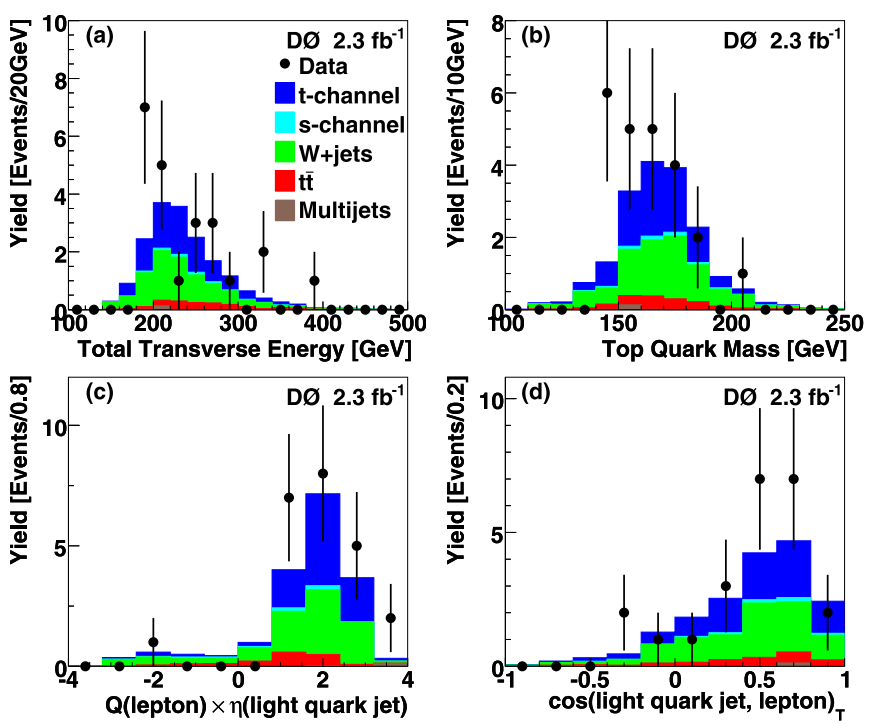

Fig. 6. $H_{T}(\mathrm{a})$, reconstructed top quark mass (b), light quark jet pseudorapidity multiplied by lepton charge (c), and $t$-channel top quark spin correlation (d, see text) for events with a ranked combination output $>0.91$. The $t$-channel and $s$-channel contributions have been normalized to their measured cross sections.

In summary, we have presented the first direct evidence of the $t$-channel mode of single top quark production using $2.3 \mathrm{fb}^{-1}$ of data at the D0 experiment. We measure a $t$-channel cross section of $3.14_{-0.80}^{+0.94} \mathrm{pb}$ and a $s$-channel cross section of $1.05 \pm 0.81 \mathrm{pb}$. The measured cross sections are consistent with the SM expected values. The observed $t$-channel signal corresponds to an excess over the predicted background with a significance of $4.8 \sigma$.

\section{Acknowledgements}

We thank the staffs at Fermilab and collaborating institutions, and acknowledge support from the DOE and NSF (USA); CEA and CNRS/IN2P3 (France); FASI, Rosatom and RFBR (Russia); CNPq, FAPERJ, FAPESP and FUNDUNESP (Brazil); DAE and DST (India); Colciencias (Colombia); CONACyT (Mexico); KRF and KOSEF (Korea); CONICET and UBACyT (Argentina); FOM (The Netherlands); STFC and the Royal Society (United Kingdom); MSMT and GACR (Czech Republic); CRC Program, CFI, NSERC and WestGrid Project (Canada); BMBF and DFG (Germany); SFI (Ireland); The Swedish Research Council (Sweden); CAS and CNSF (China); and the Alexander von Humboldt Foundation (Germany).

\section{References}

[1] V.M. Abazov, et al., DØ Collaboration, Phys. Rev. Lett. 103 (2009) 092001.

[2] T. Aaltonen, et al., CDF Collaboration, Phys. Rev. Lett. 103 (2009) 092002.

[3] N. Cabibbo, Phys. Rev. Lett. 10 (1963) 531;

M. Kobayashi, T. Maskawa, Prog. Theor. Phys. 49 (1973) 652.

[4] T. Tait, C.-P. Yuan, Phys. Rev. D 63 (2001) 014018.

[5] V.M. Abazov, et al., DØ Collaboration, Phys. Rev. Lett. 100 (2008) 211803.

[6] V.M. Abazov, et al., DØ Collaboration, Phys. Lett. B 641 (2006) 423.

[7] T. Aaltonen, et al., CDF Collaboration, Phys. Rev. Lett. 103 (2009) 041801.

[8] M. Hosch, et al., Phys. Rev. D 56 (1997) 5725.

[9] V.M. Abazov, et al., DØ Collaboration, Phys. Rev. Lett. 99 (2007) 191802.

[10] T. Aaltonen, et al., CDF Collaboration, Phys. Rev. Lett. 102 (2009) 151801.

[11] C.R. Chen, F. Larios, C.P. Yuan, Phys. Lett. B 631 (2005) 126.

[12] E. Boos, L. Dudko, T. Ohl, Eur. Phys. J. C 11 (1999) 473.

[13] A.P. Heinson, A.S. Belyaev, E.E. Boos, Phys. Rev. D 56 (1997) 3114.

[14] V.M. Abazov, et al., DØ Collaboration, Phys. Rev. Lett. 101 (2008) 221801.

[15] V.M. Abazov, et al., DØ Collaboration, Phys. Rev. Lett. 102 (2009) 092002.

[16] N. Kidonakis, Phys. Rev. D 74 (2006) 114012.

[17] V.M. Abazov, et al., DØ Collaboration, Phys. Rev. Lett. 98 (2007) 181802.

[18] V.M. Abazov, et al., DØ Collaboration, Phys. Rev. D 78 (2008) 012005. 
[19] V.M. Abazov, et al., DØ Collaboration, Nucl. Instrum. Methods Phys. Res. A 565 (2006) 463.

[20] T. Scanlon, Ph.D. thesis, Imperial College, University of London, FERMILABTHESIS-2006-43 (2006).

[21] E. Boos, et al., Phys. At. Nucl. 69 (2006) 1317;

E. Boos, et al., CompHEP Collaboration, Nucl. Instrum. Methods Phys. Res. A 534 (2004) 250.

[22] Z. Sullivan, Phys. Rev. D 70 (2004) 114012.

[23] J.M. Campbell, R. Frederix, F. Maltoni, F. Tramontano, Phys. Rev. Lett. 102 (2009) 182003.

[24] M.L. Mangano, et al., JHEP 0307 (2003) 001. We used ALPGEN version 2.05.

[25] T. Sjöstrand, et al., arXiv:hep-ph/0308153, 2003. We used PYTHIA version 6.323

[26] J. Pumplin, et al., JHEP 0207 (2002) 012;

D. Stump, et al., JHEP 0310 (2003) 046.

[27] R. Brun, F. Carminati, CERN Program Library Long Writeup W5013, 1993 (unpublished). We used GEANT version 3.21.13.

[28] N. Kidonakis, R. Vogt, Phys. Rev. D 68 (2003) 114014. At $m_{t}=170 \mathrm{GeV}$ $\sigma(p \bar{p} \rightarrow t \bar{t}+X)=7.91 \mathrm{pb}$.
[29] J.M. Campbell, R.K. Ellis, Phys. Rev. D 65 (2002) 113007, We used MCFM version 5.1 .

[30] L. Breiman, et al., Classification and Regression Trees, Wadsworth, Stamford, 1984

[31] J.A. Benitez, Ph.D. thesis, Michigan State University, FERMILAB-THESIS-2009-31 (2009).

[32] D. Gillberg, Ph.D. thesis, Simon Fraser University, FERMILAB-THESIS-2009-20 (2009).

[33] R.M. Neal, Bayesian Learning for Neural Networks, Springer-Verlag, New York, 1996.

[34] A. Tanasijczuk, Ph.D. thesis, Universidad de Buenos Aires, in preparation.

[35] V.M. Abazov, et al., DØ Collaboration, Nature 429 (2004) 638

[36] M. Pangilinan, Ph.D. thesis, Brown University, in preparation.

[37] I. Bertram, et al., FERMILAB-TM-2104 (2000).

[38] J. Alwall, et al., Eur. Phys. J. C 49 (2007) 791.

[39] C. Amsler, et al., Particle Data Group, Phys. Lett. B 667 (2008) 1.

[40] G. Mahlon, S.J. Parke, Phys. Rev. D 55 (1997) 7249.

[41] Q.H. Cao, et al., Phys. Rev. D 72 (2005) 094027. 